\title{
Screening Algorithm for BK Virus-Associated Nephropathy Using Sequential Testing of Urinary Cytology: A Probabilistic Model Analysis
}

\author{
Maggie K.M. Ma ${ }^{a} \quad$ Anskar Y.H. Leung ${ }^{a}$ Kin Yee Lo ${ }^{b}$ Weng In Liog \\ Hoi Wong Chanc Ivy Wong ${ }^{d}$ Anthony K.C. Hau ${ }^{e}$ Chun Hay Tam ${ }^{f}$ \\ Andrew K.M. Wong ${ }^{b}$ Un I. Kuok ${ }^{g}$ Ka Foon Chau ${ }^{c}$ Samuel K.S. Fung ${ }^{d}$ \\ Tsz Hoi Kwan ${ }^{\text {e Sunny S.H. Wong }}{ }^{f}$ Sydney C.W. Tang ${ }^{a}$
}

${ }^{a}$ Department of Medicine, University of Hong Kong, Queen Mary Hospital, ${ }^{b}$ Department of Medicine and Geriatrics, Kwong Wah Hospital, ' Department of Medicine, Queen Elizabeth Hospital, dDepartment of Medicine and Geriatrics, Princess Margaret Hospital, e Department of Medicine and Geriatrics, Tuen Mun Hospital, and ${ }^{\mathrm{f}}$ Department of Medicine and Geriatrics, United Christian Hospital, Hong Kong, SAR, and 9 Division of Nephrology, Centro Hospitalar Conde de São Januário, Macao, SAR, China

\section{Key Words}

BK virus-associated nephropathy $\cdot$ Kidney transplantation · Polymerase chain reaction · Screening · Urine cytology

\footnotetext{
Abstract

Background: Incorporating urinary cytology in BK virus (BKV) screening algorithm potentially reduces the screening cost for BK viral nephropathy. We aimed to evaluate the test performances and screening cost of sequential 2-stage screening consisting of urine cytology followed by BKV serum quantitative polymerase chain reaction (PCR). Methods: Ninety-five kidney transplant recipients who had BKV serum quantitative $P C R /$ urine cytology tested and verified with histopathology (the reference gold standard) were included. A probabilistic model was constructed to evaluate the test performance and screening cost of 2-stage screening, and was compared with screening with urine cytology or serum viral load alone. Results: At a viral load threshold of $\geq 10^{4}$ copies/ $\mathrm{ml}$, the sensitivity and specificity of quantitative PCR alone
}

were $83 \%$ (95\% Cl 69-96) and 91\% (95\% Cl 83-97), respectively. The sensitivity and specificity of urine cytology alone were $91 \%(95 \% \mathrm{Cl} 79-100)$ and $74 \%(95 \% \mathrm{Cl} 60-91)$, respectively. Sequential 2-stage screening resulted in loss in sensitivity but a net gain in specificity (viral load threshold $\geq 10^{4}$ copies/ml - sensitivity, 75\% (95\% Cl 60-91); specificity, $98 \%$ (95\% Cl 95-99)). Two-stage screening also had superior positive predictive value and is cost effective when BKV-associated nephropathy prevalence is below $94 \%$. Conclusions: Our study had demonstrated a favorable test performance and cost efficiency of 2-stage BKV screening.

C) 2016 S. Karger AG, Basel

\section{Introduction}

BK virus-associated nephropathy (BKVAN) occurs in $1-10 \%$ of renal transplant recipients $[1,2]$. An increasing prevalence has been noted in recent years, and this trend has been correlated with the use of newer and more po-

\section{KARGER}

E-Mail karger@karger.com

www.karger.com/ajn
(C) 2016 S. Karger AG Basel

$0250-8095 / 16 / 0426-0410 \$ 39.50 / 0$
Professor Sydney C.W. Tang, MBBS, PhD

Yu Professor in Nephrology, Division of Nephrology, Department of Medicine Queen Mary Hospital, University of Hong Kong

Hong Kong (China)

E-Mail scwtang@hku.hk 
tent immunosuppressive agents [3]. BKVAN is mostly diagnosed late in the advanced stage of the disease when irreversible damage has already occurred, leading to graft loss in $40-60 \%$ of the affected individuals [3, 4]. Hence, routine screening of BKV infection is now recommended to allow early detection of BKV replication and prompt reduction of immunosuppressant at earlier stages of the disease $[5,6]$.

Screening serum viral load alone with polymerase chain reaction (PCR) test has been shown to have satisfactory test performance and is the recommended screening tool according to the KDIGO guidelines [5]. The current American Society of Transplantation guidelines have also supported screening BKV viremia with or without prior BKV viremia screening [6]. However, the high screening cost is the major obstacle for implementing universal serum BK viral load screening in post-kidney transplant recipients.

Detection of BK viremia usually precedes the development of BK viremia or BKVAN. However, a staged approach measuring $B K$ viral load in urine followed by serum with PCR test was not cost-effective when compared with BKV serum PCR test alone [7]. Urine cytology for decoy cell is a surrogate marker of BK viremia. The test is inexpensive. Its negative predictive value (NPV) to rule out BKVAN is almost $100 \%[8,9]$. Sequential screening with urine cytology followed by BKV serum quantitative PCR potentially reduces screening cost. Nevertheless, the impact of such sequential test combination on the test performance characteristic has never been evaluated.

In this study, we aimed to compare the test performance of urine cytology, BKV serum quantitative PCR and 2-stage screening (consisting of urine cytology followed by BKV serum quantitative PCR). The screening costs of various screening methods were also evaluated.

\section{Subjects and Methods}

\section{Study Participants}

This was a retrospective analysis including 7 renal centers in Hong Kong, SAR, and Macau, SAR. This study was conducted in accordance with the Declaration of Helsinki and Declaration of Istanbul, and the study protocol was approved by the Institution Review Board/Ethics Committee. Kidney transplant recipients who had BKV serum quantitative PCR or urine cytology tested between 2006 and 2014 were evaluated, and only tests that had been verified with histopathology of the allografts were included in this study. Recorded baseline data included recipient characteristics such as age, gender, causes of end-stage kidney disease, transplantation type (categorized as deceased donor and living donor transplantation), immunological risk factors (such as the number of human leukocyte antigen (HLA) mismatches and the number of renal transplantations), immunosuppressant regime and posttransplant complications such as history of acute rejection.

\section{$B K V$ Test}

Serum BK viral load by quantitative PCR was carried out in Queen Mary Hospital by a real-time PCR using the Light Cycler 480 System II. Specimen collection and processing, sequences of the quantitative PCR primers and TaqMan probe (targeting the BKV VP1 gene), amplification and quality assurance followed the established protocol [10]. Its use in local renal transplant patients has been verified. It had been demonstrated that the BK viral load in asymptomatic patients is quantifiably low $\left(<3 \log _{10}\right.$ copies $\left./ \mathrm{ml}\right)$ and patients with biopsy-proven BKVAN had levels in the range of $6 \log _{10}$ copies/ml [11]. Urine cytology was performed by pathologists in individual hospitals. Smears stained by Papanicolaou method were assessed for the presence of decoy cells [12]. For indeterminate cases, immunostaining for polyoma large-T antigen (anti-SV40) will also be used. All the BKV tests would be paired with pathological assessment of kidney allograft biopsies which were the reference standards for diagnosing BKVAN. Diagnosis of BKVAN was based on viral cytopathic effect and confirmed by immunostaining of renal tissue for polyoma large- $\mathrm{T}$ antigen (antiSV40) [13].

\section{Statistical Analysis}

Demographic and transplant characteristics of BKVAN patients were compared with non-BKVAN patients. Comparisons between groups were performed by the chi-square statistic for categorical data and the Student $t$ test for continuous data. The receiver operator curve (ROC) for BKV serum quantitative PCR in reference to biopsy-proven BKVAN was presented. Test sensitivity and specificity of urine cytology and serum viral load at different thresholds were determined. As positive predictive value (PPV) and NPV are not fixed characteristics of screening tests and vary with population prevalence of BKVAN, we determined these post-test probabilities with the Bayes' formula [14]. The PPV and NPV of various BKV screening protocols were presented using a range of BKV prevalence rates. There was no routine BKV screening in our centers. Therefore, a probabilistic model was constructed, using the sensitivity and specificity of urine cytology and BKV PCR derived from the current cohort, to estimate the test characteristics of 2-stage screening (fig. 1). In the probabilistic model, patients were assumed to undergo urine cytology test, and only those patients who had urine decoy cell detected would be subjected to BKV serum quantitative PCR testing. Positive tests were defined as cases that have both urine decoy cell AND serum BK viral load above the cutoff threshold. Negative cases were defined as cases that have either no urine decoy cell or positive urine decoy but serum BK viral load below the cutoff threshold. Sensitivity, specificity, PPV and NPV were derived from the probabilistic model, and $95 \%$ CIs of all screening strategies were determined by bootstrap resampling method [15]. We also conducted a cost analysis to compare the screening cost of BKV screening with 2-stage screening and screening with serum BK viral load alone, using a hypothetical cohort of 1,000 transplant patients. All analyses were performed using SPSS version 16.0 (SPSS Inc., Chicago, Ill., USA) and R statistical software 3.0.2 (R Foundation for Statistical Computing, 2013), with $\mathrm{p}$ values $<0.05$ considered statistically significant. 


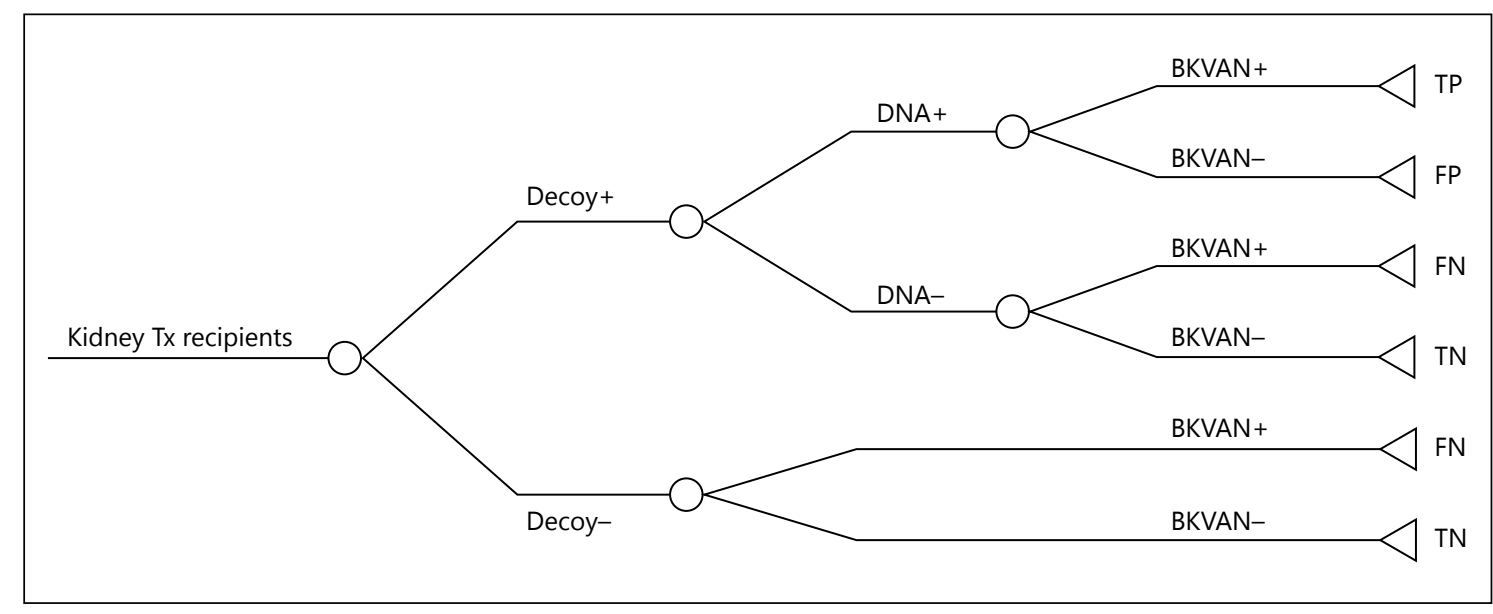

Fig. 1. Probabilistic model of the 2-stage sequential screening algorithm using urine cytology followed by BKV serum quantitative PCR. Structure of the decision tree. TP = True positive; $\mathrm{TN}=$ true negative; $\mathrm{FP}=$ false positive; $\mathrm{FN}$ = false negative.

\section{Results}

\section{Study Population}

A total of 192 post-kidney transplantation patients had BKV serum quantitative PCR performed during the study period. Of the 192 cases, 95 had matched allograft biopsy to verify the BKV test results and were included in the analysis. For the remaining 97 patients who were excluded, most of them were low risk for any pathological diagnosis. They had serum BK quantitative PCR because of transient allograft dysfunction that spontaneously improved $(n=68)$, for screening of newly transplanted patients $(n=19)$, successful completion of BKVAN treatment $(n=3)$ and unknown reason $(n=7)$.

Twenty-nine out of 95 patients (30.5\%) had biopsyproven BKVAN. The pathological diagnosis of the 66 non-BKVAN cases included acute rejection/borderline change ( $n=29)$, glomerulonephritis $(n=9)$, interstitial fibrosis and tubular atrophy $(\mathrm{n}=15)$ and others $(\mathrm{n}=$ 13). Table 1 summarized the baseline characteristics of kidney transplant recipients with and without BKVAN. The average age was $45.4 \pm 13.3$ and $45.8 \pm 12.6$ years for BKVAN and non-BKVAN patients, respectively. The distribution of primary kidney disease, transplantation types, HLA mismatches, use of induction therapies and rejection rate were similar between both the groups. There was a significantly higher proportion of BKVAN patients using the tacrolimus-based immunosuppressant (non-BKVAN: 22 (33.3\%) vs. BKVAN: 20 (69.0\%), $\mathrm{p}<0.001)$. Among the BKVAN patients, 17 cases $(60.7 \%)$ were diagnosed within the first post-transplant year. The remaining $10(35.7 \%)$ and 1 cases (3.6\%) were diagnosed in $1-5$ years and $\geq 5$ years post transplant, respectively.

\section{Sensitivity and Specificity of Urine Cytology, BK}

Serum Viral Load and 2-Stage Screening

Of the 95 patients who had BKV serum quantitative PCR test, 24 (36.4\%) non-BKVAN patients and 1 (3.4\%) BKVAN patient had negative test. Thirty-six (54.5\%) non-BKVAN patients and $4(13.8 \%)$ BKVAN patients had BK viremia detected at level $<10^{4}$ copies $/ \mathrm{ml}$. Six (9.1\%) non-BKVAN patients and 24 (82.8\%) BKVAN patients had BK viral load $\geq 10^{4}$ copies $/ \mathrm{ml}$.

The ROC curve evaluating the test performance of BKV serum quantitative PCR is shown in figure 2. The area under curve was estimated to be 0.92 (95\% CI $0.83-$ $0.98)$. The sensitivity and specificity of BKV serum quantitative PCR test at various cutoff thresholds were summarized in table 2. At a threshold of $\geq 10^{4}$ copies $/ \mathrm{ml}$, the test sensitivity and specificity for BKVAN were 83\% (95\% CI 69-96) and 91\% (95\% CI 83-97), respectively. Increasing the cutoff threshold would increase the specificity but reduce the sensitivity of the test.

Sixty-nine patients (non-BKVAN: $\mathrm{n}=46$; BKVAN: $\mathrm{n}=23$ ) had urine cytology performed. Decoy cell is present in $12(26.1 \%)$ non-BKVAN and 21 (91.3\%) BKVAN patients, respectively. The sensitivity and specificity of urine cytology for BKVAN were 91\% (95\% CI 79-100) and $74 \%$ (95\% CI 60-91), respectively (table 2).

Table 2 summarized the sensitivity and specificity of 2 -stage screening at various serum viral load cutoff 
Table 1. Characteristics of kidney transplant recipients with and without BKVAN

\begin{tabular}{|c|c|c|c|}
\hline & $\begin{array}{l}\text { No BKVAN } \\
(n=66)\end{array}$ & $\begin{array}{l}\text { With BKVAN } \\
(\mathrm{n}=29)\end{array}$ & $\mathrm{p}$ value \\
\hline Age at transplantation, years & $45.8 \pm 12.6$ & $45.4 \pm 13.3$ & 0.89 \\
\hline Male gender, n (\%) & $46(69.7)$ & $18(62.1)$ & 0.47 \\
\hline Number of transplant, n (\%) & & & 0.90 \\
\hline 1 & $61(92.4)$ & $27(93.1)$ & \\
\hline 2 & $5(7.6)$ & $2(6.9)$ & \\
\hline Primary kidney disease, n (\%) & & & 0.44 \\
\hline Chronic glomerulonephritis & $16(24.2)$ & $11(37.9)$ & \\
\hline Diabetic nephropathy & $19(28.8)$ & $4(13.8)$ & \\
\hline Hypertensive nephrosclerosis & $3(4.5)$ & $2(6.9)$ & \\
\hline Adult polycystic kidney disease & $4(6.1)$ & $2(6.9)$ & \\
\hline Unknown & $21(31.8)$ & $10(34.5)$ & \\
\hline Others & $3(4.5)$ & 0 & \\
\hline Deceased donor, n (\%) & $61(92.4)$ & $28(96.6)$ & 0.45 \\
\hline HLA mismatch, $\mathrm{n}(\%)$ & & & 0.62 \\
\hline 0 & $3(4.5)$ & 0 & \\
\hline $1-4$ & $19(28.8)$ & $10(34.5)$ & \\
\hline $5-6$ & $7(10.6)$ & $4(13.8)$ & \\
\hline Unknown & $37(56.1)$ & $15(51.7)$ & \\
\hline Use of induction therapy, $\mathrm{n}(\%)$ & & & 0.30 \\
\hline No induction & $43(65.2)$ & $23(79.3)$ & \\
\hline IL-2 $\mathrm{R}$ antagonist & $11(16.7)$ & $4(13.8)$ & \\
\hline ATG & $12(18.2)$ & $2(6.9)$ & \\
\hline Immunosuppressant regimen, $\mathrm{n}(\%)$ & & & $<0.001$ \\
\hline Prednisolone/CSA \pm anti-metabolite & $43(65.2)$ & $5(17.2)$ & \\
\hline Prednisolone/Tac \pm anti-metabolite & $22(33.3)$ & $20(69.0)$ & \\
\hline Prednisolone/mTORi \pm 3rd agent & $1(1.5)$ & $4(13.8)$ & \\
\hline Duration of transplant at time of BKV testing, years, $\mathrm{n}(\%)$ & & & 0.05 \\
\hline$<1$ & $28(42.4)$ & $17(60.7)$ & \\
\hline $1-5$ & $22(33.3)$ & $10(35.7)$ & \\
\hline$>5$ & $16(24.2)$ & $1(3.6)$ & \\
\hline History of rejection, $\mathrm{n}(\%)$ & $18(27.3)$ & $9(31.0)$ & 0.71 \\
\hline Viremia, copies/ml & & & $<0.001$ \\
\hline 0 & $24(36.4)$ & $1(3.4)$ & \\
\hline$<10^{4}$ & $36(54.5)$ & $4(13.8)$ & \\
\hline $10^{4}-<10^{5}(>4 \log )$ & $4(6.1)$ & $7(24.1)$ & \\
\hline$>10^{5}-<10^{6}(>5 \log )$ & $2(3.0)$ & $17(58.6)$ & \\
\hline Number of urine cytology tests, $\mathrm{n}(\%)$ & 46 & 23 & \\
\hline Decoy cell presence & $12(26.1)$ & $21(91.3)$ & $<0.005$ \\
\hline
\end{tabular}

$\mathrm{IL}-2 \mathrm{R}$ antagonist $=$ Interleukin 2 receptor antagonist; $\mathrm{CSA}=$ cyclosporin $\mathrm{A} ; \mathrm{Tac}=$ tacrolimus; $\mathrm{mTORi}=$ mTOR inhibitor.

thresholds. Sequential testing with urine cytology followed by BKV serum quantitative PCR resulted in loss in sensitivity but a net gain in specificity, compared to either of the tests used alone. At viral load threshold $\geq 10^{4}$ copies/ml, the sensitivity and specificity of the 2-stage screening algorithm were 75\% (95\% CI 60-91) and 98\% (95\% CI 95-99). Increasing the cutoff threshold would increase the specificity but reduce the sensitivity.
PPV and NPV of Urine Cytology, BKV Serum

Quantitative PCR and 2-Stage Screening

PPV and NPV are not fixed characteristics of the test and depend on the prevalence of the disease in the population tested and the validity of the test (sensitivity and specificity). Figure 3 presented the PPV and NPV of various BKV screening protocols according to a range of $\mathrm{BKV}$ prevalence rates. Urine cytology had the lowest PPV irrespective of BKVAN prevalence rates. BKV serum 
Table 2. Test performances of urinary cytology, serum BKV quantitative PCR and 2-stage screening

\begin{tabular}{lll}
\hline & Sensitivity, \% (95\% CI) & Specificity, \% (95\% CI) \\
\hline Urine decoy cell & $91(79-100)$ & $74(60-91)$ \\
Serum BKV DNA $>1 \times 10^{3} \mathrm{copies} / \mathrm{ml}$ & $93(82-100)$ & $82(72-91)$ \\
Serum BKV DNA $>1 \times 10^{4} \mathrm{copies} / \mathrm{ml}$ & $83(69-96)$ & $91(83-97)$ \\
Serum BKV DNA $>1 \times 10^{5} \mathrm{copies} / \mathrm{ml}$ & $59(41-77)$ & $97(92-100)$ \\
Two-stage $\left(\right.$ BKV DNA $\left.>1 \times 10^{3} \mathrm{copies} / \mathrm{ml}\right)$ & $85(71-97)$ & $95(91-98)$ \\
Two-stage (BKV DNA $\left.>1 \times 10^{4} \mathrm{copies} / \mathrm{ml}\right)$ & $75(60-91)$ & $98(95-99)$ \\
Two-stage $\left(\right.$ BKV DNA $>1 \times 10^{5}$ copies $\left./ \mathrm{ml}\right)$ & $54(35-72)$ & $99(98-100)$ \\
\hline
\end{tabular}

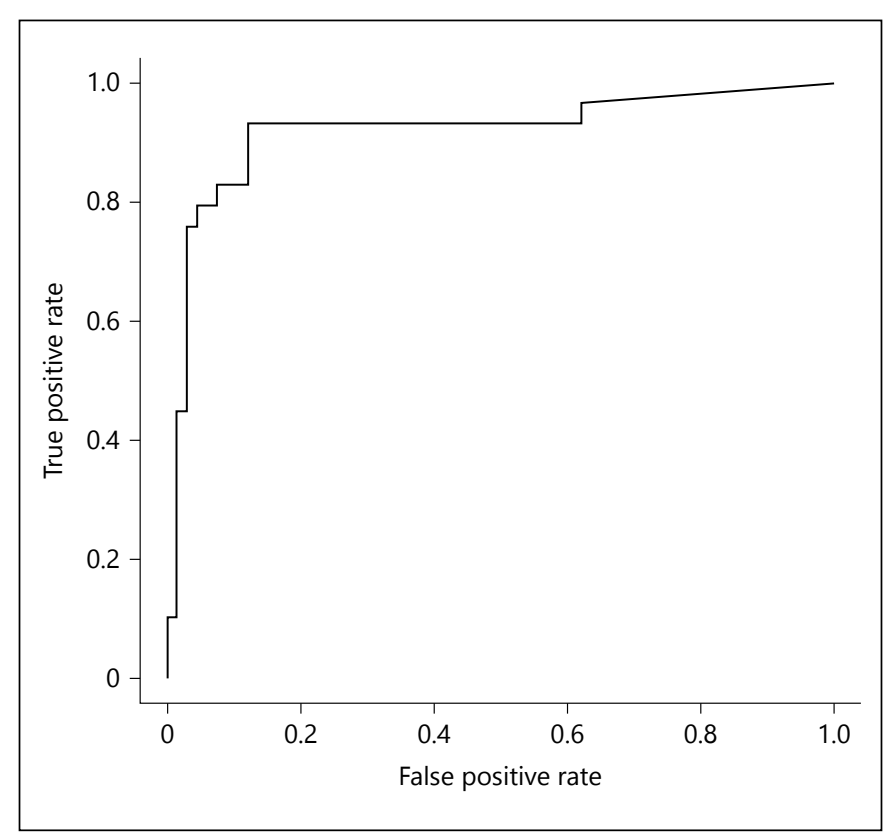

Fig. 2. ROCs for BKV serum quantitative PCR. The AUC 0.92 (95\% CI 0.83-0.98).

quantitative PCR has higher PPV than urine cytology. Increasing the viral load threshold would improve the PPV of serum quantitative PCR. Two-stage screening using viral load threshold $\geq 10^{4}$ copies $/ \mathrm{ml}$ had superior PPV when compared with either urine cytology or BKV serum quantitative PCR alone (fig. 3a).

BKV serum quantitative PCR threshold $\leq 10^{3}$ copies/ $\mathrm{ml}$ had the best NPV irrespective of BKVAN prevalence rate. Increasing the viral load threshold would reduce the NPV of serum quantitative PCR. Two-stage screening using viral load threshold $\geq 10^{4}$ copies $/ \mathrm{ml}$ had non-inferior NPV when compared with either urine cytology or BKV serum quantitative PCR alone if BKVAN prevalence ranged from 1 to $10 \%$, which is the commonly reported range of BKVAN prevalence (fig. 3b, shaded area) [1,2].

\section{Cost Analyses}

In-house BKV DNA assay is available in Queen Mary Hospital at a cost of around US $\$ 150$ per assay. The average screening cost of urine cytology is around US\$20. All cost estimation included both staff cost and consumables. We assume yearly BKV screening in a hypothetical cohort of 1,000 kidney transplant recipients. The average screening cost for serum BK viral load testing alone would be US\$150,000. The screening cost of 2-stage screening test would vary with BKVAN prevalence. With increasing prevalence, there would be increasing cases with decoy cell detected that need further testing with serum viral load, hence increasing the screening cost (fig. 4). The 2-stage screening costs less than BKV serum quantitative PCR alone when BKVAN prevalence is low and only confers an increased screening cost when BKVAN prevalence exceeds $94 \%$.

\section{Discussions}

Compared with BKV screening with urine cytology alone and BK serum viral quantitative PCR alone, sequential 2-stage screening had improved specificity at the cost of reduced sensitivity. The prevalences of BKVAN were commonly reported between 1 and $10 \%$. In these range of prevalence rates, 2-stage screening had superior PPV and non-inferior NPV. In addition, 2-stage screening is cost effective when BKVAN prevalence is below $94 \%$.

The management of BKV infection remains a significant problem, and an effective anti-viral treatment is still lacking. Therefore, the cornerstone of management hinges upon early detection of BKV reactivation and cautious reduction of immunosuppressant. BKV screening with urine decoy cell analysis is inexpensive and has high sensitivity. However, its low PPV may result in high false positive rates and unnecessary biopsy trigger. Detecting 


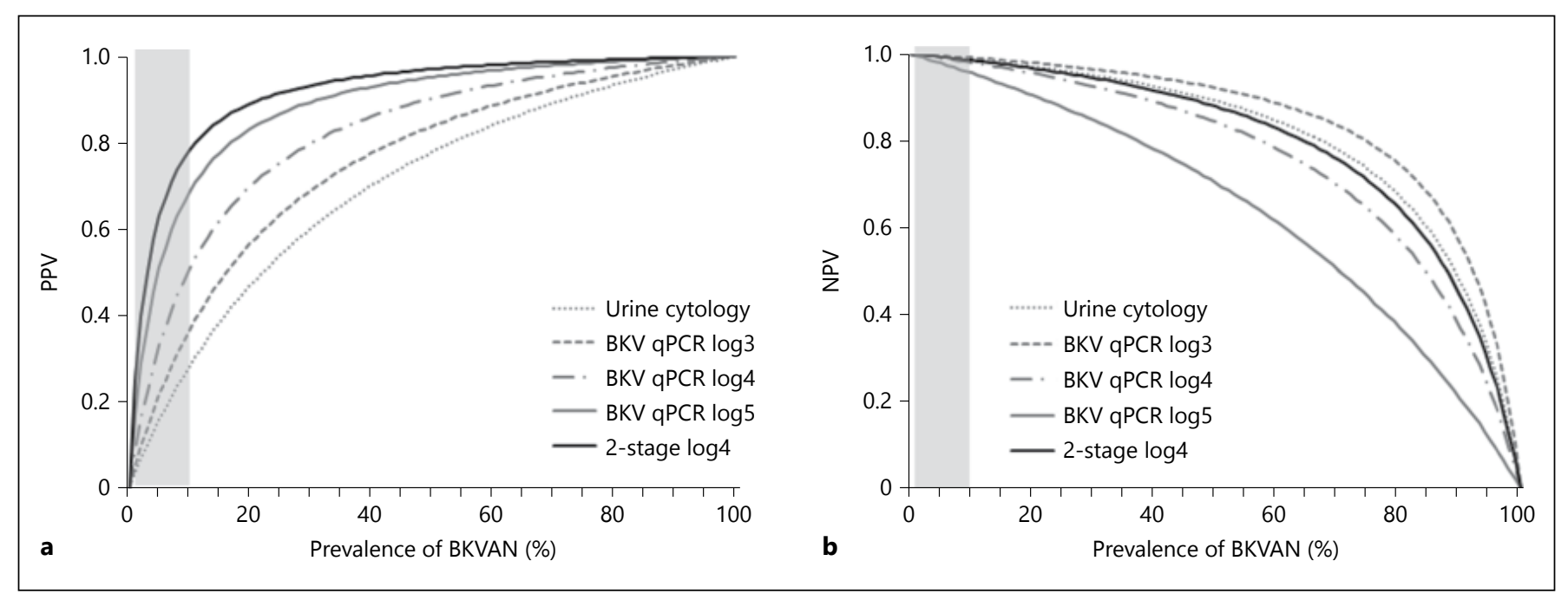

Fig. 3. The PPV (a) and NPV (b) of urinary cytology, serum BKV quantitative PCR and 2-stage screening according to BKVAN prevalence. Shaded area indicates the commonly reported range of BKVAN prevalence.

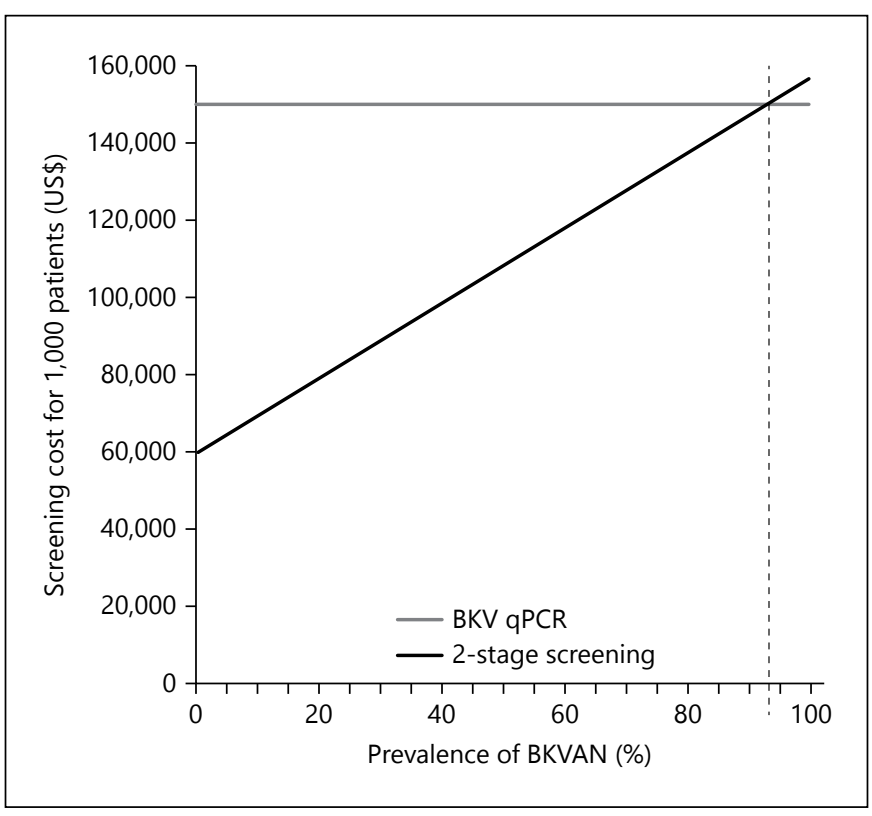

Fig. 4. Screening cost of 2-stage screening and BKV serum quantitative PCR alone. The graph presents the screening cost according to BKVAN prevalence in a hypothetical cohort of 1,000 kidney transplant recipients (dash line indicate the threshold value when the cost of 2-stage screening exceed serum quantitative PCR alone).

serum viral load alone with PCR test has been shown to have excellent NPV of almost $100 \%$ and quantitative PCR would improve the PPV from 40 to $80 \%$ [4, 16-18]. KDIGO guideline now suggests screening all renal transplant recipients with quantitative nucleic acid testing
(NAT) and reducing the dose of immunosuppressant when BKV plasma NAT is persistently $>10^{4}$ copies $/ \mathrm{ml}$ [5]. However, the high screening cost is the major obstacle for adopting it as a routine screening strategy. Combining decoy cell and viremia simultaneously with 'AND' and 'OR' combination for BKVAN screening has been evaluated [18]. Only comparable sensitivity was achieved in the 'OR' combination (when compared with testing serum viral load alone) and PPV was compromised in the 'AND' combination.

From our knowledge, this is the first study to explicitly evaluate the test performance of sequential screening algorithm with urine cytology followed by BKV serum quantitative PCR. Urine cytology is a useful screening test to rule out BKVAN. At low BKVAN prevalence, significant proportion of low risk patients would be screened out by urine cytology. As urine cytology has high sensitivity, the chance of screened failure (i.e., false negative) would also be low. The remaining individuals who have urinary decoy cell and need further serum viral load testing would be of higher risk of BKVAN (i.e., higher prevalence) and hence higher PPV would be achieved in the second stage BKV serum quantitative PCR.

In addition to the satisfactory test performance, such sequential 2-stage screening algorithm is cost effective. Chakera et al. [19] also reported saving of approximately $£ 135,000$ over 2 years in BKV screening using decoy cell followed by serum viral load for cases with sustained decoy cell, compared with routine surveillance by quantitative PCR alone. A Canadian group had created a compre- 
hensive cost-effective model and demonstrated that centers with modest or high rate $(>3 \%)$ of BKVAN could benefit most from routine BKV screening consisting of combined blood and urine tests [20]. However, in this study, there was no fixed BKV screening protocol, and a wide range was explored in their sensitivity analysis. Smith et al. [21] have performed a cost analysis for sequential screening test. It has been illustrated that reduction in immunosuppressant covers the cost of screening for polyoma viral infection. Compared with serum viral load screening alone, we also demonstrated 2-stage screening is cost effective when prevalence of BKVAN is below $94 \%$.

Our study has both strengths and potential limitations. Unlike many other studies that did not have histological reference test and only inferred diagnosis in the screened negative cases, both BKVAN and non-BKVAN cases included in our study have been verified with histopathology. We also illustrated the impact of changing the viral load cutoff threshold on the test characteristics in different screening protocols. The limitations of our study include the small sample size and retrospective design. We do not have a routine BKV screening protocol. The samples included in our cohort were those with biopsy for cause and PCR testing. Not all subjects in the cohort had urine cytology testing. In order to minimize biased estimate of the test characteristic of 2-stage screening, we constructed a probabilistic model and derived the test characteristic parameters using the sensitivity and specificity of urine cytology and serum BKV quantitative PCR, which are fixed characteristics and not influenced by the study cohort characteristics (such as disease prevalence).
On the other hand, PPV and NPV are post-test probabilities that are significantly influenced by the prevalence of disease in the population tested. Thus, we presented them in a range of BKVAN prevalence. Implementing BKV screening and pre-emptive reduction of immunosuppressant in cases with sustained viremia detected by 2 -stage screening protocol has both positive and negative sides. This potentially improves graft survival and reduces graft failure from BKVAN but at the tradeoff of increased rejection risk. Further study to delineate the benefit and risk will be warranted. In our study, only screening cost was included in the cost analysis. Cost utility analysis that evaluates the direct/indirect costs would also be necessary.

To conclude, sequential testing of BKV with urine cytology followed by quantitating serum viral load would improve test specificity and has the highest PPV to identify BKVAN cases and non-inferior NPV to rule out BKVAN at lower screening cost. The favorable test performance characteristics and cost efficiency suggested that 2-stage screening would be the BKV screening test of choice, particularly in centers that cannot afford routine screening with serum PCR alone. Although urine cytology is historically an 'old standard' for investigating suspected BKVAN, which is no longer performed in many hospitals including those in the US, our results suggest that this inexpensive assay should be revitalized.

\section{Disclosure Statement}

None to declare.

\section{References}

1 Hirsch HH: Polyomavirus BK nephropathy: a (re-)emerging complication in renal transplantation. Am J Transplant 2002;2:25-30.

2 Hirsch HH, Steiger J: Polyomavirus BK. Lancet Infect Dis 2003;3:611-623.

3 Hariharan S: BK virus nephritis after renal transplantation. Kidney Int 2006;69:655662.

4 Kuppachi S, Thomas B, Kokko KE: BK virus in the kidney transplant patient. Am J Med Sci 2013;345:482-488.

5 KDIGO clinical practice guideline for the care of kidney transplant recipients. Am J Transplant 2009;9(suppl 3):S1-S155.

6 Hirsch HH, Randhawa P; AST Infectious Diseases Community of Practice: BK polyomavirus in solid organ transplantation. Am J Transplant 2013;13(suppl 4):179-188.
7 Laskin BL, Goebel J: Cost-efficient screening for BK virus in pediatric kidney transplantation: a single-center experience and review of the literature. Pediatr Transplant 2010;14: 589-595.

8 Huang G, Chen WF, Wang CX, Fei JG, Deng SX, Qiu J, Chen LZ: Noninvasive tool for the diagnosis of polyomavirus $\mathrm{BK}$-associated nephropathy in renal transplant recipients. Diagn Microbiol Infect Dis 2013;75:292-297.

9 Nickeleit V, True K, Detwiler R, Kozlowski T, Singh H: Risk assessment for polyomavirus nephropathy using urine cytology and the detection of decoy cells: cheap and efficient. Transplantation 2012;94:e42-e44; author reply e45.

10 Leung AY, Suen CK, Lie AK, Liang RH, Yuen KY, Kwong YL: Quantification of polyoma
BK viruria in hemorrhagic cystitis complicating bone marrow transplantation. Blood 2001;98:1971-1978.

11 Chan GC, Leung AY, Wong AS, Chan KW, Kwong YL, Lai KN, Tang SC: Quantification of BK viral load in asymptomatic renal allograft recipients. Ren Fail 2012;34:550-554.

12 Ranzi AD, Prolla JC, Keitel E, Brackmann R, Kist R, dos Santos G, Bica CG: The role of urine cytology for 'decoy cells' as a screening tool in renal transplant recipients. Acta Cytol 2012;56:543-547.

13 Masutani K, Shapiro R, Basu A, Tan H, Wijkstrom M, Randhawa P: The Banff 2009 Working Proposal for polyomavirus nephropathy: a critical evaluation of its utility as a determinant of clinical outcome. Am J Transplant 2012;12:907-918. 
14 Sonnenberg A: We only see what we already know - a modified bayes' formula to explain inherent limitations of diagnostic tests. Med Hypotheses 2004;63:759-763.

15 Efron B, Tibshirani RJ: An Introduction to the Bootstrap. New York, Chapman and Hall/ CRC, 1994.

16 Myint TM, Turner RM, Craig JC, Cross NB, Kable K, Nankivell BJ, Chapman JR, Webster AC, O'Connell P, Dwyer DE, Jeoffreys N, Roger SD, Wong G: Test performance characteristics of quantitative nucleic acid testing for polyomaviruses in kidney and kidneypancreas transplant recipients. Clin Transplant 2013;27:E571-E579.
17 Westervelt JD, Alexander BD, Costa SF, Miller SE, Howell DN, Smith SR: Detection of BK polyomavirus after kidney transplantation: a comparison of urine electron microscopy with plasma polymerase chain reaction. Clin Transplant 2013;27:E42-E48.

18 Nankivell BJ, Renthawa J, Jeoffreys N, Kable K, O'Connell PJ, Chapman JR, Wong G, Sharma RN: Clinical utility of urinary cytology to detect BK viral nephropathy. Transplantation 2015;99:1715-1722.
19 Chakera A, Dyar OJ, Hughes E, Bennett S, Hughes D, Roberts IS: Detection of polyomavirus $\mathrm{BK}$ reactivation after renal transplantation using an intensive decoy cell surveillance program is cost-effective. Transplantation 2011;92:1018-1023.

20 Kiberd BA: Screening to prevent polyoma virus nephropathy: a medical decision analysis. Am J Transplant 2005;5:2410-2416.

21 Smith F, Panek R, Kiberd BA: Screening to prevent polyoma virus nephropathy in kidney transplantation: a cost analysis. Am J Transplant 2009;9:2177-2179. 